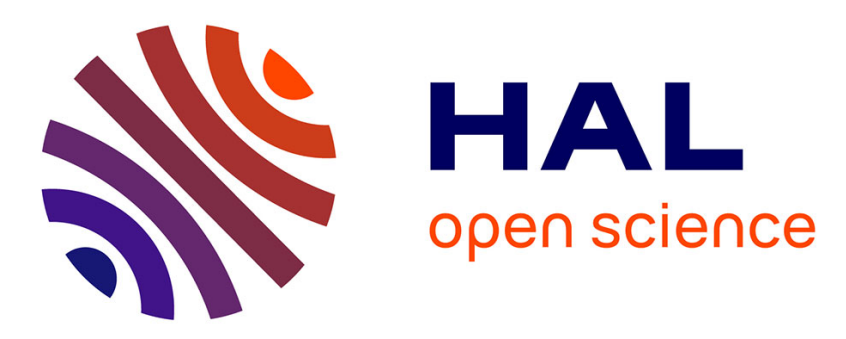

\title{
Controllability and Observability of Leader-Based Multi-agent Systems
}

Rogelio Lozano, Mark W. Spong, Jose Alfredo Guerrero, Nikhil Chopra

\section{To cite this version:}

Rogelio Lozano, Mark W. Spong, Jose Alfredo Guerrero, Nikhil Chopra. Controllability and Observability of Leader-Based Multi-agent Systems. Proceedings of the 47th IEEE Conference on Decision and Control, CDC 2008, 2008, Cancun, Mexico. hal-00448757

\section{HAL Id: hal-00448757 https://hal.science/hal-00448757}

Submitted on 20 Jan 2010

HAL is a multi-disciplinary open access archive for the deposit and dissemination of scientific research documents, whether they are published or not. The documents may come from teaching and research institutions in France or abroad, or from public or private research centers.
L'archive ouverte pluridisciplinaire HAL, est destinée au dépôt et à la diffusion de documents scientifiques de niveau recherche, publiés ou non, émanant des établissements d'enseignement et de recherche français ou étrangers, des laboratoires publics ou privés. 


\title{
Controllability and Observability of Leader-Based Multi-agent Systems
}

\author{
R.Lozano, M.W. Spong, J. A. Guerrero, N. Chopra
}

\begin{abstract}
In this work we analyze the controllability and observability properties of several interconnection configurations such as the chain topology and cyclic topology as well as combinations of these two topologies. A leader/follower control strategy is proposed to control the center of mass of the multiple agent system. It is shown that the trajectory tracking for a multi-agent system converges to the constant input reference given only to the leader. Also, it is shown that choosing an appropriated gain, the agents achieve consensus for constant input reference.
\end{abstract}

Index Terms-Multi-agent coordination, Trajectory tracking, Formation Control, Controllability, Observability.

\section{INTRODUCTION}

Multiple agent coordination as well as multiple spacecraft flying in formation has been intensively investigated during the last decade. Coordination control of multiple aerial, ground or underwater vehicles has important applications. They include the transport of heavy or large loads, search and rescue operations, space or ocean exploration, etc.

Different approaches have been proposed in the literature for coordinating multiple robot systems. There are mainly three approaches: Leader/Follower, Virtual Structure and Behavioral Control.

In the leader/follower architecture, one agent is designated as leader while the others are designated as followers which should track the leader. Leader/follower approaches are described in: [1], [2]. The virtual structure approach considers every agent as an element of a larger structure [3]. Finally the behavioral control in [4] and [5] is based on the decomposition of the main control goal into tasks or behaviors. This approach also deals with collision avoidance, flock centering, obstacle avoidance and barycenter.

Consensus algorithms allow the coordination of velocities and/or positions of multiple agents. They have been the object of extensive analysis and development [6], [7] and[8]. Trajectory tracking of flocks has been recently studied in [9] and [10].

A natural way to analyze the relationship and communication between agents is using directed or undirected graphs. Every node in a graph is considered as an agent which can have information exchange with all or several agents. In [7], [8], and [11], the authors use algebraic graph theory in order to model the information exchange between vehicles. By using this technique several control strategies have been developed. [9] presents a new strategy for consensus in multiagent systems with a time varying reference. Several cases

R. Lozano and J.A. Guerrero are with the Heudiasyc Laboratory,CNRS 6599 UTC, France. E-mail: rlozano@hds.utc.fr. M.W. Spong is with the Information Trust Institute at the UIUC, USA. N. Chopra is with the Department of Mechanical Engineering at the University of Maryland, USA. are presented, such as: all agents have access to the reference, several agents have access to the reference, etc. The analysis presented assumes that each agent evolution is represented by a first order integrator.

In [12] and [13] an analysis of multiple agent coordination using a passivity approach to decompose the system into two passive subsystems is presented. The first subsystem called "shape" maintains the formation of the group of agents while the second subsystem called "lock" represents the translational dynamics of the group. In [13], the convergence of velocity and relative position of the agents via passive decomposition is shown.

A bilateral teleoperation approach has been used in [10] to teleoperate a group of agents. The authors provide results to achieve a bilateral teleoperation one-to-many (i.e. one master and many slaves in a leader/follower achitecture). The center of mass is used as a virtual master robot which is used to coordinate the slave robots. Trajectory tracking is also considered using an input to state stability analysis.

Most of the papers in literature dealing with multiple agent coordination consider fully actuated agents capable of movement in all directions. Some represent the agents by single integrators and some other by double integrators. A state of the art in consensus algorithms can be found in [6].

In this paper we propose a passive approach for multiplevehicle coordination and flock trajectory tracking control. The study is mainly focused on two configurations: cyclic and chain configurations of information exchange between agents. The control strategy is composed of two terms. The first control term is used for agent consensus and the second control term is used for achieving a desired position of the formation center of mass. We use the output synchronization control in [14] for agent coordination. This type of strategy is such that the control input for each agent depends only on the information coming from its neighbors. Tracking of the center of mass is achieved by using a full state feedback control on the leader. Since the leader is not assumed to have direct information from all the agents, the state is observed from the input and output of the leader. We therefore require observability and controllability (or at least detectability and stabilizability) of the agents network from the leader input and output. Observability and controllability of agent formations is studied for the case of ring and chain topologies using a coordinating controller. Controllability and observability of leader-based multi-agent coordination has been studied in [15]-[18]. Here, the authors provide sufficient conditions for multi-agent coordination controllability and observability using multiple leaders and how interconnection graph topology determines controllability and observability 
properties.

The paper is organized as follows: Section 2 introduces the background and preliminaries on information graph theory. Section 3 presents the dynamic model of the proposed configurations. Section 4 presents necessary conditions to satisfy the controllability and observability of the multiagent system. Flock trajectory tracking control based on the centroid of the system is also presented. Section 5 is devoted to simulation results. Conclusions are given in section 6 .

\section{PRELIMINARIES}

A multi-agent dynamic system can be modelled as a group of dynamical systems which has a information exchange topology represented by information graphs. A graph $\mathcal{G}$ is a pair $\mathcal{G}(\mathcal{N}, \mathcal{E})$ consisting of a set of nodes $\mathcal{N}=$ $\left\{n_{i}: n_{i} \in \mathcal{N}, \forall i=1, \ldots, n\right\}$ together with their interconnections $\mathcal{E}$ on $\mathcal{N}$ [6]. Each pair $\left(n_{1}, n_{2}\right)$ is called an edge $e \in \mathcal{E}$. An undirected graph is one where nodes $i$ and $j$ can get information from each other. In a digraph, the $i^{\text {th }}$ node can get information from the $j^{\text {th }}$ node but not necessarily viceversa. We can think of the information exchange between agents as an undirected graph but also as a digraph which implies a more complicated problem. A graph is connected if for every pair $\{x, y\}$ of distinct vertices there is a path from $x$ to $y$. A connected graph allows the communication between all agents through the network. A graph is said to be balanced if its in-degree (number of communication links arriving at the node) is equal to its out-degree (number of communication links leaving the node).

\section{CONTROLLABILITY AND OBSERVABILITY OF INTERCONNECTIONS}

We will study the controllability and observability of interconnections using a coordinating control strategy. We will assume that the agents are represented by double integrators. We will first consider the case of three agents and study the controllability and observability of the system using only the input and output of a single agent. It is shown that for the cyclic topology the system is observable and controllable from any agent. It also shown that for the chain topology with undirected communication, the system is controllable and observable for agents 1 and 3 but only stabilizable and detectable for agent 2 .

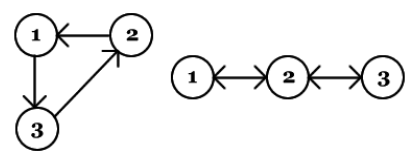

Fig. 1. Information flow configuration: a) Cyclic topology (left), b) Chain topology (right).

\section{A. Cyclic topology}

In the case of 3 agents, the cyclic topology with input and output on the first agent, is represented as

$$
\left[\begin{array}{l}
\ddot{x}_{1} \\
\ddot{x}_{2} \\
\ddot{x}_{3}
\end{array}\right]=\left[\begin{array}{ccc}
-1 & 1 & 0 \\
0 & -1 & 1 \\
1 & 0 & -1
\end{array}\right]\left[\begin{array}{l}
\dot{x}_{1} \\
\dot{x}_{2} \\
\dot{x}_{3}
\end{array}\right]+\left[\begin{array}{l}
1 \\
0 \\
0
\end{array}\right] u_{1}
$$

$$
y_{1}=\left[\begin{array}{lll}
1 & 0 & 0
\end{array}\right]\left[\begin{array}{c}
\dot{x}_{1} \\
\dot{x}_{2} \\
\dot{x}_{3}
\end{array}\right]
$$

Let $\mathcal{O}$ and $\mathcal{C}$ be the observability and controllability matrices. It is clear that the system is observable since det $\mathcal{O}=1$. Also the system is controllable since $\operatorname{det} \mathcal{C}=-1$.

\section{B. Chain topology. Input and output on agent 1 .}

In the chain topology with input and output of the first agent, the system is represented by

$$
\begin{gathered}
{\left[\begin{array}{c}
\ddot{x}_{1} \\
\ddot{x}_{2} \\
\ddot{x}_{3}
\end{array}\right]=\left[\begin{array}{ccc}
-1 & 1 & 0 \\
1 & -2 & 1 \\
0 & 1 & -1
\end{array}\right]\left[\begin{array}{l}
\dot{x}_{1} \\
\dot{x}_{2} \\
\dot{x}_{3}
\end{array}\right]+\left[\begin{array}{l}
1 \\
0 \\
0
\end{array}\right] u_{1}} \\
y_{1}=\left[\begin{array}{lll}
1 & 0 & 0
\end{array}\right]\left[\begin{array}{l}
\dot{x}_{1} \\
\dot{x}_{2} \\
\dot{x}_{3}
\end{array}\right]
\end{gathered}
$$

The system is observable since $\operatorname{det} \mathcal{O}=1$. The system is also controllable since $\operatorname{det} \mathcal{C}=1$. Due to symmetry, the same result holds for agent 3 .

\section{Chain topology. Input and output on agent 2.}

Let us consider the same chain configuration as before but considering this time the input and output of agent 2 .

$$
\begin{gathered}
{\left[\begin{array}{l}
\ddot{x}_{1} \\
\ddot{x}_{2} \\
\ddot{x}_{3}
\end{array}\right]=\left[\begin{array}{ccc}
-1 & 1 & 0 \\
1 & -2 & 1 \\
0 & 1 & -1
\end{array}\right]\left[\begin{array}{l}
\dot{x}_{1} \\
\dot{x}_{2} \\
\dot{x}_{3}
\end{array}\right]+\left[\begin{array}{l}
0 \\
1 \\
0
\end{array}\right] u_{2}} \\
y_{1}=\left[\begin{array}{lll}
0 & 1 & 0
\end{array}\right]\left[\begin{array}{l}
\dot{x}_{1} \\
\dot{x}_{2} \\
\dot{x}_{3}
\end{array}\right]
\end{gathered}
$$

The system is not observable since $\operatorname{det} \mathcal{O}=0$. Note however that we assumed that agent 2 measures all the state.

The system is not controllable since det $\mathcal{C}=0$. Let $w_{1}^{T}, w_{2}^{T}$ and $w_{3}^{T}$ be the eigenvectors of the Laplacian in (4), see (21). Premultiplying (4) by $w_{1}^{T}, w_{2}^{T}, w_{3}^{T}$, we obtain

$$
\begin{array}{ccc}
\ddot{x}_{1}+\ddot{x}_{2}+\ddot{x}_{3} & = & u_{2} \\
\ddot{x}_{1}-2 \ddot{x}_{2}+\ddot{x}_{3} & = & -3\left(\dot{x}_{1}-2 \dot{x}_{2}+\dot{x}_{3}\right)+u_{2} \\
\ddot{x}_{1}-\ddot{x}_{3} & = & -\left(\dot{x}_{1}-\dot{x}_{3}\right)
\end{array}
$$

Equations (4) can also be rewritten as

$$
\begin{array}{ccc}
\ddot{x}_{2} & = & -2 \dot{x}_{2}+\left(\dot{x}_{1}+\dot{x}_{3}\right)+u_{2} \\
\ddot{x}_{1}+\ddot{x}_{3} & = & 2 \dot{x}_{2}-\left(\dot{x}_{1}+\dot{x}_{3}\right) \\
\ddot{x}_{1}-\ddot{x}_{3} & = & -\left(\dot{x}_{1}-\dot{x}_{3}\right)
\end{array}
$$

The last equation represents a stable uncontrollable mode. It follows that $\dot{x}_{1}-\dot{x}_{3}=e(0) \exp ^{-t}$ with $e(0)=\dot{x}_{1}(0)-$ $\dot{x}_{3}(0)$. Thus defining $z=\dot{x}_{1}+\dot{x}_{3}$, (6) can be reduced to:

$$
\begin{array}{ccc}
\ddot{x}_{2} & = & -2 \dot{x}_{2}+z+u_{2} \\
\dot{z} & = & 2 \dot{x}_{2}-z
\end{array}
$$

The reduced system is controllable since $\operatorname{det} \mathcal{C}=2$. Also, the reduced system is observable since $\operatorname{det} \mathcal{O}=1$. 


\section{General case}

The examples above suggest that the interconnection of agents using the coordinating control strategy leads to systems that are controllable and observable from the input and output of every agent or that are at least stabilizable and detectable. We will prove next that this is true in the general case when the system is represented by

$$
\begin{aligned}
\ddot{x} & =-\mathcal{L} \dot{x}+b u \\
y & =c^{T} \dot{x}
\end{aligned}
$$

where $\mathcal{L}$ is the Laplacian matrix having the following properties:

1) $\mathcal{L}$ has a single eigenvalue at $0, \lambda_{1}(\mathcal{L})=0$ with right eigenvector $w_{1}^{T}=\left[\begin{array}{llll}1 & 1 & \cdots & 1\end{array}\right]$, i.e. $\mathcal{L} w_{1}=0$.

2) The remaining eigenvalues have all positive real part, i.e. $\operatorname{Re}\left[\lambda_{i}(\mathcal{L})\right]>0$ and $\mathcal{L} w_{i}=\lambda_{i} w_{i}$ for $i=2, . . n$, and $w_{i} \in R^{n}$. If $\mathcal{L}$ is symmetric then the remaining eigenvalues are all positive, i.e. $\lambda_{i}(\mathcal{L})>0$.

We assume that the information exchange graph is balanced. Let us assume also that in the coordinating controller the gains multiplying the signals in between agents are all equal to 1 . For the $i-t h$ row of $\mathcal{L}$, the entries $l_{i j}=-1$ for $i \neq j$ correspond to the gains multiplying the signals from other agents coming to agent $i$. For the $i-t h$ column of $\mathcal{L}$, the entries $l_{j i}=-1$ for $i \neq j$ correspond to the gains multiplying the signals going out of agent $i$ towards the other agents. We then have the following property.

3) $w_{1}$ defined above is also the left eigenvalue of $\mathcal{L}$ corresponding to the eigenvalue 0 , i.e. $w_{1}^{T} \mathcal{L}=0$.

Let us study the controllability and observability of the system from the input and output of agent $k, i . e . c^{T}=$ $b^{T}=\left[\begin{array}{lllllll}0 & \cdots & 0 & 1 & 0 & \cdots & 0\end{array}\right]$ where only the $k-t h$ element is different from zero.

Proposition 1: Consider the multiple agent system whose evolution is described by (7). This system is unobservable if there exist a right eigenvector $\omega_{i}$ of $\mathcal{L}$ such that $c^{T} \omega_{i}=0$.

Proof: Let $\omega_{1}$ be the right eigenvector of $\mathcal{L}$ corresponding to the zero eigenvalue. Note that $c^{T} \omega_{1} \neq 0$, and thus, the mode corresponding to $\left(\lambda_{1}, w_{1}\right)$ is observable. If $c^{T} w_{i}=0$ for some $i=2, . . n$, then the system will have non observable modes, but such modes will be asymptotically stable, i.e., they will converge to zero, because $\operatorname{Re}\left[\lambda_{i}(\mathcal{L})\right]>0$ for $i=2, \ldots, n$.

Proposition 2: Consider the multiple agent system whose evolution is described by (7). This system is uncontrollable if there exist an eigenvector $v_{i}$ of $\mathcal{L}^{T}$ such that $v_{i}^{T} b=0$.

Proof: Note that $\mathcal{L}$ and $\mathcal{L}^{T}$ have the same eigenvalues. Let $v_{i}$ be the right eigenvectors of $\mathcal{L}^{T}$ (or the left eigenvectors of $\mathcal{L}$ ), i.e. $\mathcal{L}^{T} v_{i}=\lambda_{i} v_{i}$ or $v_{i}^{T} \mathcal{L}=\lambda_{i} v_{i}^{T}$. Pre-multiplying $(7)$ by $v_{i}^{T}$ we get

$$
v_{i}^{T} \ddot{x}=-v_{i}^{T} \mathcal{L} \dot{x}+v_{i}^{T} b u
$$

or

$$
v_{i}^{T} \ddot{x}=-\lambda_{i} v_{i}^{T} \dot{X}+v_{i}^{T} b u
$$

If $v_{i}^{T} b=0$ for some $i$, then

$$
\frac{d}{d t}\left(v_{i}^{T} \dot{x}\right)=-\lambda_{i}\left(v_{i}^{T} \dot{x}\right)
$$

which means that the corresponding mode is not controllable. Nevertheless, for $i=2, \ldots, n$ such mode is asymptotically stable and converges to zero. Recall that for $i=1$ we have $v_{1}=w_{1}$ and therefore $v_{1}^{T} b \neq 0$. Thus the mode corresponding to $\left(\lambda_{1}, v_{1}\right)$ is controllable.

Lemma 1: Center of mass of multi-agent system (7) corresponds to controllable and observable modes.

\section{E. The cyclic topology in the general case}

In this section it will be proved that the cyclic topology is controllable and observable in the general case. The laplacian matrix of the cyclic topology is the following

$$
-\mathcal{L}=\left[\begin{array}{ccccc}
-1 & 1 & & & \\
& -1 & 1 & & \\
& & \ddots & \ddots & \\
& & & -1 & 1 \\
1 & & & & -1
\end{array}\right]
$$

and $c=b^{T}=\left[\begin{array}{lllll}0 & \ldots & 1 & \cdots & 0\end{array}\right]$ where only the kth element is different from zero. For simplicity, this system will be rewritten as

$$
\begin{aligned}
\ddot{x} & =-\mathcal{L}_{r} \dot{x}+b u_{l} \\
y_{l} & =c^{T} \dot{x}
\end{aligned}
$$

1) Observability: The system will be non observable if there exists a vector $v$ such that

$$
v^{T} b=0
$$

and

$$
\mathcal{L}_{r} v=\lambda v
$$

with $v^{T}=\left[\begin{array}{llll}v_{1} & v_{2} & \cdots & v_{n}\end{array}\right]$ and a real $\lambda$.

For $\lambda=0, v^{T}=\left[\begin{array}{llll}1 & 1 & \cdots & 1\end{array}\right]$ and thus $v^{T} b \neq 0$ which is a contradiction.

For $\lambda \neq 0$, it follows from (10) that $v_{1}=0$. In view of the structure of $\mathcal{L}_{r}$ in (11) it follows that $v_{2}=0$. Iterating it follows that $v=0$. We conclude that the system is observable.

2) Controllability: The system will be non controllable if there exists a vector $v$ such that

$$
v^{T} b=0
$$

and

$$
v^{T} \mathcal{L}_{r}=\lambda v^{T}
$$

with $v^{T}=\left[\begin{array}{llll}v_{1} & v_{2} & \cdots & v_{n}\end{array}\right]$ and a real $\lambda$.

For $\lambda=0$, it follows from (12) that $v_{1}=0$. In view of (13) it follows that $v_{2}=0$. Iterating it follows that $v=0$.

For $\lambda \neq 0$, since $v_{1}=0$ and in view of the structure of $\mathcal{L}_{r}$ in (13) it follows that $v_{n}=0$. Substituting in (13) gives $v_{n-1}=0$. Iterating it follows $v=0$. We conclude that the system is controllable. 


\section{F. The chain topology in the general case}

In this section it will be proved that the chain topology is controllable and observable in the general case. The laplacian matrix of the chain topology is the following

$$
-\mathcal{L}=\left[\begin{array}{ccccc}
-1 & 1 & & & \\
1 & -2 & 1 & & \\
& \ddots & \ddots & \ddots & \\
& & 1 & -2 & 1 \\
& & & 1 & -1
\end{array}\right]
$$

and $c=b^{T}=\left[\begin{array}{lllll}0 & \cdots & 1 & \cdots & 0\end{array}\right]$ where only the kth element is different from zero. For simplicity, this system will be rewritten as

$$
\begin{aligned}
\ddot{x} & =-\mathcal{L}_{c} \dot{x}+b u_{l} \\
y_{l} & =c^{T} \dot{x}
\end{aligned}
$$

1) Controllability: The system will be non controllable if there exists a vector $v$ such that

$$
v^{T} b=0
$$

and

$$
v^{T} \mathcal{L}_{c}=\lambda v^{T}
$$

with $v^{T}=\left[\begin{array}{llll}v_{1} & v_{2} & \cdots & v_{n}\end{array}\right]$ and a real $\lambda$.

For $\lambda=0$, since $v_{1}=0$, from (16) it follows that $v_{2}=0$. Iterating it follows that $v=0$.

For $\lambda \neq 0$, in view of the structure of $\mathcal{L}_{c}$ in (14) it follows from (16) that $v_{2}=0$. Iterating it follows that $v=0$. We conclude that the system is controllable.

2) Observability: Given that $\mathcal{L}_{c}=\mathcal{L}_{c}^{T}$ and $b=c^{T}$, the system is also observable.

\section{G. Combinations of chain and cyclic topologies}

In this section we show that a network of agents obtained by appropriately combining the cyclic and chain topologies is controllable and observable. Let us consider the following topology

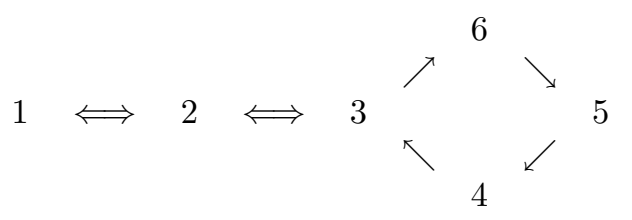

Considering the input and output of agent 1 , the state space representation is given by

$$
\begin{aligned}
\ddot{x} & =-\mathcal{L}_{c o} \dot{x}+b u_{1} \\
y_{1} & =c^{T} \dot{x}
\end{aligned}
$$

where

$$
-\mathcal{L}_{c o}=\left[\begin{array}{cccccc}
-1 & 1 & & & & \\
1 & -2 & 1 & & & \\
& 1 & -2 & 1 & & \\
& & & -1 & 1 & \\
& & & & -1 & 1 \\
& & 1 & & & -1
\end{array}\right]
$$

and $c=b^{T}=\left[\begin{array}{llll}1 & 0 & \cdots & 0\end{array}\right]$.
1) Controllability: The system will be non controllable if there exists a vector $v$ such that

$$
v^{T} b=0
$$

and

$$
v^{T} \mathcal{L}_{c o}=\lambda v^{T}
$$

with $v^{T}=\left[\begin{array}{llll}v_{1} & v_{2} & \cdots & v_{6}\end{array}\right]$ and $\lambda$ real.

Case $\lambda=0$. In this case the only vector satisafing (18) is $v^{T}=\left[\begin{array}{llll}1 & 1 & \cdots & 1\end{array}\right]$ which implies that $v^{T} b \neq 0$.

Case $\lambda \neq 0$. In view of the structure of $\mathcal{L}_{c o}$ in (17) it follows that $v_{1}=0 \Longrightarrow v_{2}=0$. Similarly it follows that $v_{3}=0, v_{6}=0, v_{4}=0$ and $v_{5}=0$.

We conclude that the system is controllable.

2) Observability: The system will be non observable if there exists a vector $v$ such that

$$
v^{T} b=0
$$

and

$$
\mathcal{L}_{c o} v=\lambda v
$$

with $v^{T}=\left[\begin{array}{llll}v_{1} & v_{2} & \cdots & v_{6}\end{array}\right]$ and a real $\lambda$.

Case $\lambda=0$. The only vector satisfying (20) is $v^{T}=$ $\left[\begin{array}{cccc}1 & 1 & \cdots & 1\end{array}\right]$ and thus $v^{T} b \neq 0$ which is a contradiction.

Case $\lambda \neq 0$. In view of the structure of $\mathcal{L}_{c o}$ in (18) and since $v_{1}=0$, it follows that $v_{2}=0$. Iterating it follows that $v=0$. We conclude that the system is observable.

Remark 1: In view of the structure of $\mathcal{L}_{c o}$ in (18) and the arguments described above, it follows that the controllability and the observability properties are preserved if the chain and the cyclic topologies in (17) have arbitrary length.

\section{H. Simple configurations that are either non controllable or non observable}

In order to help characterizing the configurations that are both controllable and observable, we present in this section a series of illustrative simple configurations that are not in the form (17) and that fail to be controllable and observable. We will denote by $\mathcal{L}, \mathcal{C}_{i}, \mathcal{O}_{i}$ the Laplacian, the controllability and observability matrices respectively when using the input and output of the $i-t h$ agent.

1) Example 1:

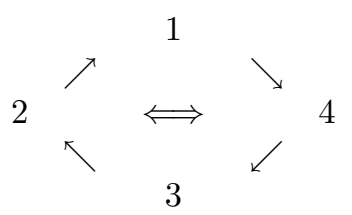

Note that $\operatorname{det} \mathcal{C}_{1}=\operatorname{det} \mathcal{C}_{2}=0$. We have that $\operatorname{det} \mathcal{O}_{1}=$ $\operatorname{det} \mathcal{O}_{2}=0$

2) Example 2:

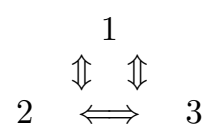

Note that $\operatorname{det} \mathcal{C}_{1}=\operatorname{det} \mathcal{O}_{1}=0$ 
3) Example 3:

$$
2 \longleftarrow \begin{array}{r}
1 \\
\downarrow \\
\longleftarrow
\end{array} 34
$$

$\operatorname{det} \mathcal{C}_{1}=0, \operatorname{det} \mathcal{O}_{1}=1, \operatorname{det} \mathcal{C}_{2}=-1, \operatorname{det} \mathcal{O}_{2}=0, \operatorname{det} \mathcal{C}_{3}=$ $0, \operatorname{det} \mathcal{O}_{3}=0, \operatorname{det} \mathcal{C}_{4}=1, \operatorname{det} \mathcal{O}_{4}=-1$ Controllability and observability from agent 4 can also be proved as it was done for (17).

4) Example 4:

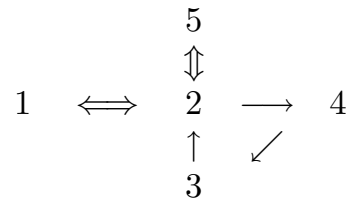

$\operatorname{det} \mathcal{C}_{1}=0, \operatorname{det} \mathcal{O}_{1}=0$

5) Example 5:

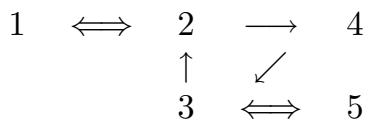

$\operatorname{det} \mathcal{C}_{1}=-1, \operatorname{det} \mathcal{O}_{1}=0$

\section{FORMATION LEADER TRACKING}

In this section we introduce a coordination control for flock tracking. We first study the case of three agents and then we extend the method to the general case.

\section{A. Eigenvalues and eigenvectors of the system}

Consider the case of three agents in chain topology described in (3). The eigenvalues of $A$ in (3) are $0,-1,-3$. Their corresponding eigenvectors are

$$
\begin{aligned}
& w_{1}^{T}=\left[\begin{array}{ccc}
1 & 1 & 1
\end{array}\right] \\
& w_{2}^{T}=\left[\begin{array}{lll}
1 & 0 & -1 \\
1 & -2 & 1
\end{array}\right] \\
& w_{3}^{T}=\left[\begin{array}{ll}
T \\
\text { T }
\end{array}\right]
\end{aligned}
$$

Premultiplying (3) by $w_{1}^{T}, w_{2}^{T}, w_{3}^{T}$ above we obtain

$$
\begin{array}{ccc}
\ddot{x}_{1}+\ddot{x}_{2}+\ddot{x}_{3} & = & u_{1} \\
\ddot{x}_{1}-\ddot{x}_{3} & = & -\left(\dot{x}_{1}-\dot{x}_{3}\right)+u_{1} \\
\ddot{x}_{1}-2 \ddot{x}_{2}+\ddot{x}_{3} & = & -3\left(\dot{x}_{1}-2 \dot{x}_{2}+\dot{x}_{3}\right)+u_{1}
\end{array}
$$

Define $\dot{x}_{C M}=\frac{1}{3} \sum_{i=1}^{3} \dot{x}_{i}$ and its desired velocity value $\dot{x}_{C M}^{d}$. Consider the tracking control law

$$
u_{1}=3 k s a t\left\{\dot{x}_{C M}^{d}-\dot{x}_{C M}\right\}
$$

where $\operatorname{sat}(\cdot)$ represents the saturation function and $k$ is a positive gain. Note that $\dot{x}_{C M}$ is not directly measurable by the leader (agent 1), but the state can be observed from the input and output of agent 1 . Defining $e_{C M}=\dot{x}_{C M}^{d}-\dot{x}_{C M}$ and introducing (23) into (22) we get

$$
\begin{array}{ccc}
\ddot{x}_{C M} & = & k s a t\left\{e_{C M}\right\} \\
\ddot{x}_{1}-\ddot{x}_{3} & = & -\left(\dot{x}_{1}-\dot{x}_{3}\right)+3 k s a t\left\{e_{C M}\right\} \\
\ddot{x}_{1}-2 \ddot{x}_{2}+\ddot{x}_{3} & = & -3\left(\dot{x}_{1}-2 \dot{x}_{2}+\dot{x}_{3}\right)+3 k s a t\left\{e_{C M}\right\}
\end{array}
$$

If the desired velocity value $\dot{x}_{C M}^{d}$ is constant, then $\dot{x}_{C M} \longrightarrow$ $\dot{x}_{C M}^{d}$ as $t \longrightarrow \infty$, which implies that $u_{1} \longrightarrow 0$ and $\left(\dot{x}_{1}-\right.$ $\left.\dot{x}_{3}\right),\left(\dot{x}_{1}-\dot{x}_{2}\right) \longrightarrow 0$. Notice that for small values of $k$, the convergence speed of $\dot{x}_{C M}$ is slow, but the transient in the errors $\left(\dot{x}_{1}-\dot{x}_{3}\right),\left(\dot{x}_{1}-\dot{x}_{2}\right)$ will be smaller.

From the previous analysis we can state the following lemma

Lemma 2: Consider a multi-agent system of the form (7) with coordinating control law (23). If $\dot{x}_{C M}^{d}$ is constant, then $\dot{x}_{C M} \longrightarrow \dot{x}_{C M}^{d}$ as $t \longrightarrow \infty$,which implies that $u_{1} \longrightarrow 0$ and $\left(\dot{x}_{i}-\dot{x}_{j}\right) \longrightarrow 0$.

\section{B. Formation leader tracking in the general case}

Define $\dot{x}_{C M}=\frac{1}{N} \sum_{i=1}^{N} \dot{x}_{i}$ where $N$ is the number of agents in the formation. Let $\dot{x}_{C M}^{d}$ be the desired value for $\dot{x}_{C M}$. Assume for simplicity that agent 1 is the leader, i.e. $c^{T}=$ $b^{T}=\left[\begin{array}{llll}1 & 0 & \cdots & 0\end{array}\right]$ and that the control law is

$$
u_{1}=N k s a t\left\{\dot{x}_{C M}^{d}-\dot{x}_{C M}\right\}
$$

where $\operatorname{sat}(\cdot)$ represents the saturation function and $k$ is a positive gain. Note that $\dot{x}_{C M}$ may not be directly measurable for the leader (agent 1). We assume the system is observable from the input and output of the leader. The state can therefore be observed from the input and output of agent 1. Introducing (23) into (8) we get

$$
\begin{array}{ccc}
\ddot{x}_{C M} & = & k s a t\left\{\dot{x}_{C M}^{d}-\dot{x}_{C M}\right\} \\
\frac{d}{d t}\left(v_{i}^{T} \dot{X}\right) & = & -\lambda_{i}\left(v_{i}^{T} \dot{X}\right)+v_{i}^{T} b u_{1} ; i=2, . ., N
\end{array}
$$

The modes in the last equation above are all stable. When $u_{1}=0$, these modes converge to zero which means that $\left(\dot{x}_{i}-\dot{x}_{j}\right) \longrightarrow 0$ for $i \neq j$. This property is obtained by using the coordinating control algorithm that leads to system (7). These modes are uncontrollable when $v_{i}^{T} b=0$. There is a trade-off in the choice of gain $k$ in (24). For smaller values of $k$, the speed of convergence of $\dot{x}_{C M}$ is slower, but the transient in the errors $\left(\dot{x}_{i}-\dot{x}_{j}\right)$ for $i \neq j$, will be smaller.

\section{Observer design}

Due to the nature of information flow between agents, full state is in general not available. Thus, we have developed a coordination control based on the center of mass of the multiagent system. Full state is needed in order to compute the coordination control (24). In order to obtain the full state we propose a Luenberger observer of the form:

$$
\begin{aligned}
\dot{x} & =A x-B u(\hat{x}) \\
\dot{\hat{x}} & =\bar{L} C x+(A-\bar{L} C) \hat{x}-u(\hat{x}) \\
y & =C x
\end{aligned}
$$

where $x$ is the state vector, $\hat{x}$ is the observed state vector, $\bar{L}$ is the Luenberger gain vector.

\section{SIMULATIONS}

We will consider both, the case when partial and full state of the multi-agent system is available from measurement. When the state is not available from measurement a Luenberger observer is used to estimate the state. It is shown that the multi-agent system synchronizes in velocity and 
position using the combination of coordinating and tracking control. Synchronization of the center of mass is achieved with respect to a continuous time varying reference while there is a small bias in agents synchronization with the input reference. When observer is used in the multiagent system, simulation results (Fig. 2 - Fig. 3) show the observer state convergence to the actual state which implies a convergence of the center of mass to the input reference. Agents' state also converge to the center of mass reference.

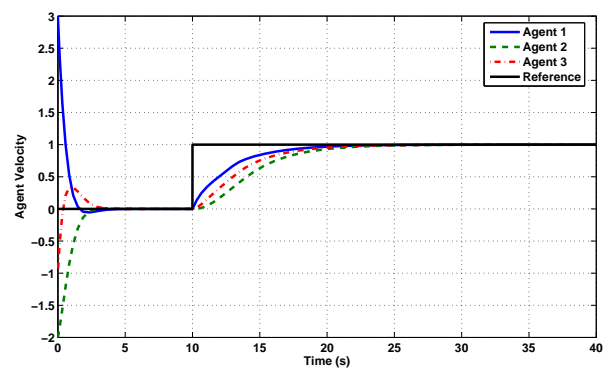

Fig. 2. Case a) Velocity consensus and tracking considering full state available for 3 -agent chain configuration.

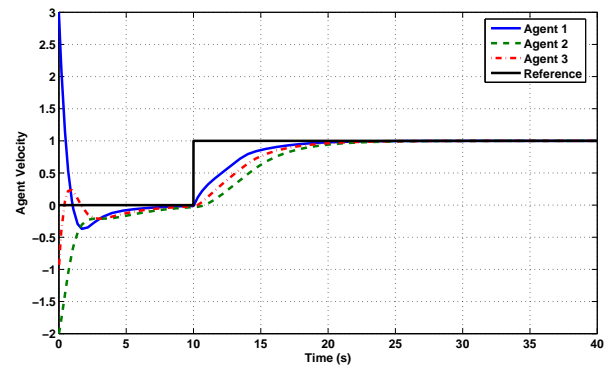

Fig. 3. Case b) Velocity consensus and tracking using Luenberger Observer for 3-agent chain configuration.

\section{CONCLUSION}

In this paper we have proposed a control method for synchronizing and tracking multiple agents in formation. We have used the coordinating control in [14] for which the input of every agent depends only on its neighbors information.

Tracking of the center of mass of the agents formation has been achieved by using state feedback control applied to the leader. Since the leader is not assumed to have direct information from all the agents, the state is observed from the input and output of the leader. This approach requires observability and controllability (or at least detectability and stabilizability) of the agents network from the leader input and output. The observability/controllability properties for chain and cyclic topologies has been studied in this paper.

It has been shown that the interconnection of agents using the coordinating control strategy and leading to systems as (7) are stabilizable and detectable from the input and output of any agent.

It has been proved that, after applying a coordinating control, the cyclic topology is observable and controllable from any agent and the chain topology is observable and controllable from the first or the last agent of the chain. A cyclic topology and a chain topology can be interconnected in cascade to obtain a new configuration that is controllable and observable provided that the input and output are taken from the first agent of the chain topology as in (17). Several simple counter-examples show that adding any extra interconnection to the cyclic topology, the chain topology or the combination of both as in (17) leads to either uncontrollable or unobservable modes. This means that the most general combination of chain and cyclic topologies that is both controllable and observable from one agent (the first agent of the chain) is the cascade interconnection of a chain topology and a cyclic topology as in (17).

\section{REFERENCES}

[1] X. Chen, A. Serrani, ISS-Based Robust Leader/Follower Trailing Control, LNCIS 336 Group Coordination and Cooperative Control, Springer-Verlag, Germany, 2006.

[2] R. Kristiansen, A. Loría, A. Chaillet, P.J. Nicklasson, Output Feedback Control of Relative Translation in a Leader-Follower Spacecraft Formation, LNCIS 336 Group Coordination and Cooperative Control, Springer-Verlag, Germany, 2006.

[3] N.E. Leaonard, E. Fiorelli, Virtual Leaders, Artificial Potentials and Coordinated Control of Groups. Proc. of the 40th IEEE Conf. on Decision and Control, 2001.

[4] F. Arrichiello, S. Chiaverini, T.I Fossen, Formation Control of Marine Vessels using the Null-Space-Based Behavioral Control, LNCIS 336 Group Coordination and Cooperative Control, Springer-Verlag, Germany, 2006.

[5] T. Balch, R.C. Arkin, Behavior-based Formation Control for multirobot teams, IEEE Trans. on Robotics and Automation, Vol. 14, No. 6, 1998.

[6] W. Ren, R.W. Beard, E.M. Atkins, Information Consensus in Multivehicule Cooperative Control, IEEE Control Systems Magazine, Vol. 27, No. 2, April 2007.

[7] H.G. Tanner, A. Jadbabaie, G.J. Pappas, Stable Flocking of Mobile Agents, Part I: Fixed Topology, Proc. of the 42nd IEEE Conference on Decision and Control, Maui, 2003

[8] H.G. Tanner, A. Jadbabaie, G.J. Pappas, Stable Flocking of Mobile Agents, Part II: Dynamic Topology, Proc. of the 42nd IEEE Conference on Decision and Control, Maui, 2003

[9] W. Ren, Consensus Seeking in Multi-vehicule Systems with a Time Varying Reference State, Proc. of the 2007 American Control Conference, N.Y., 2007.

[10] P. Hokayem, D. Stipanovic, M. Spong, Reliable Control of Multiagent Formations, IEEE Proc. of the American Control Conference, New York, 2007.

[11] R. Olfati-Saber, Flocking for Multi-Agent Dynamic Systems: Algorithms and Theory, IEEE Trans. on Automatic Control, Vol. 51, No.3, 2006.

[12] DJ. Lee, P.Y. Li, Formation and Maneuver Control of Multiple Spacecraft, IEEE Proc. of the American Control Conference, 2003.

[13] DJ. Lee, M.W. Spong, Flocking of Multiple Inertial Agents on Balanced Graph, IEEE Proc. of the American Control Conference, 2006.

[14] N. Chopra, Output Synchronization of Mechanical Systems, UIUC PhD Thesis, 2006.

[15] M. Ji, A. Muhammad, M. Egerstedt, Leader-Based Multiagent Coordination: Controllability and Optimal Control, IEEE Proc. of the American Control Conference, Minneapolis, 2006.

[16] M. Ji, M. Egerstedt, Observability and Estimation in Distributed Sensor Networks, 46th IEEE Conference on Decision and Control, New Orleans, USA, 2007.

[17] A. Rahmani, M. Mesbahi, On the Controlled Agreement Problem, IEEE Proc. of the American Control Conference, Minneapolis, 2006.

[18] A. Rahmani, M. Mesbahi, Pulling the Strings on Agreement: Anchoring, Controllability and Graph Automorphims, IEEE Proc. of the American Control Conference, New York, 2007. 